\title{
Morphosyntactic Development and Implicit Learning in Down Syndrome and Related Conditions
}

\author{
Jean Adolphe Rondal ${ }^{*}$
}

Department of Psychology, Logopedics and Education Sciences, University of Liège, B-32 Sart Tilman, 4000 Liège, Belgium

\begin{abstract}
Implicit learning of morphosyntax is attracting much interest in psycholinguistics. Limited data are available from experimental studies in people with cognitive handicap. The paper presents and discusses central aspects of the implicit learning model as it applies to morphosyntactic development in typical children and children and adolescents with cognitive handicap. Several possible reasons for the delays and difficulties usually observed in the latter are examined. Suggestions are made for further research and language intervention in cognitive handicap.
\end{abstract}

Keywords: Attentional focus, associative processes, mental representation, relational semantics, morphosyntactic patterns.

\section{INTRODUCTION}

Morphosyntax in natural languages is learned in an implicit manner in natural context through interpersonal interaction. The question has spurred much interest in psycholinguistics in recent years [1]. Theoretical suggestions have been made in transposing from experimental data on learning artificial grammars to natural morphosyntax acquisition [2]. There is no reason to believe that people with cognitive handicap do not learn the morphosyntax of their language in the same implicit way as typically developing (TD) persons albeit with delays and difficulties [3]. In the first part of the paper, an implicit learning model applying to natural morphosyntax acquisition is presented. In the second part, the available data from experimental research on language-relevant implicit learning in people with cognitive handicap are reviewed. In the third part of the paper, reasons why particular limitations in implicit learning may complicate the acquisition of morphosyntax in these people are discussed. Specific suggestions will be made regarding further research on this topic and intervention perspectives.

\section{ON EXPLAINING NATURAL MORPHOSYNTACTIC DEVELOPMENT}

The acquisition of morphosyntax in natural language remains one of the more controversial issues in psycholinguistics. By syntax, I mean the sequential organization of the words in utterances according to the normative dispositions of the language, corresponding to the pragmatic objective at the onset of the

*Address correspondence to this author at the Department of Psychology, Logopedics and Education Sciences, University of Liège, B-32 Sart Tilman, 4000 Liège, Belgium; E-mail: jeanarondal@skynet.be communicative act and the semantic matrix accounting for the relational meanings between words. Morphosyntax involves grammatical morphology which, in English, mainly corresponds to the marking of gender and number on nouns and personal pronouns, definite or indefinite reference on articles, time, aspect, and person on verbs, and agreement in number between grammatical subject and verb.

Mainstream psycholinguistics has it that language users necessarily rely on grammatical categories, formal rules, and hierarchies of notions remote from sentence surface. However, strong arguments may be levelled against this conception [4, 5]. They may be summarized as follows: (1) ordinary language users do not have conscious knowledge of these categories, rules, and hierarchies; (2) being remote from sentence surface, these notions do not seem to be learnable in natural conditions except perhaps with the help of powerful neural networks which do not belong to the natural equipment of human language learners; and (3) no genetic blueprint of universal grammar that would guide morphosyntactic development in particular languages, as advocated by representational innatism, has been found despite impressive progresses in molecular genetics over the last decades. A couple of so-called language genes have been identified (FOXP2 and CNTNAP2, both located on chromosome 7). They are involved in regulating the transcription level of a series of other genes for FOXP2, and the interconnection of cerebral areas for CNTNAP2. Mutations of these genes are associated with various language and motor pathologies such as verbal dyspraxia, dysgraphia, dysphasia, speech and language developmental delays, and a series of other conditions going from the autistic spectrum to the syndrome of Gilles de la Tourette (characterized by 
involuntary body movements and vocal productions). Stromswold [6] has reviewed genetic studies regarding language (concordance analyses of disorders in twins, adoption studies, linkage studies of familial disorders. Genetic factors account for much of the variance in language abilities among people with language disorders and some of the variance in normal people. However, nowhere is it demonstrated that these factors have a role in coding formal linguistic notions as implied by representational innatism. Rather, they are involved in neural structures important for language processing; possibly participating in the building of an operational basis for sequential ordering, arguably a central aspect of morphosyntactic processing and a task for which the left brain is anatomically well-suited [7].

\section{SURFACE TREATMENT AND IMPLICIT MORPHOSYNTACTIC LEARNING}

Recent theoretical proposals favor a direct translation of the pragmatic and semantic bases onto the surface of the utterances [5]. Neurological work suggests that human morphosyntax may be regulated by a processor relying on surface associative and sequence learning mechanisms [8, 9]. Ullman [10] has proposed a declarative/procedural model of language learning and functioning in which lexical knowledge depends on declarative (explicit) memory whereas grammar relies on another system underlying implicit procedural memory. Procedural learning plays a major role in identifying context-dependent relations between elements or events in real-time sequences. Learning occurs on an ongoing basis during multiple presentations. The knowledge acquired applies automatically to corresponding or analogically related material. Although operated by different mechanisms, the two systems of memory appear to share the same encoding factors and rely on similar perceptual processes [11]. Double dissociations between the systems have been documented in developmental and adult-onset language disorders (e.g., brain lesions, Alzheimer disease, Parkinson disease, Korsakoff syndrome, acute depressive states). Implicit memory involves the frontal, parietal and superior temporal cortices of the left brain, the left-basal ganglia (most importantly the striatum with the caudate nucleus and the putamen), and the right-neocerebellar structures. Explicit memory depends on the integrity of the prefrontal cortex, the caudate nucleus at the basis of the brain, and relies on a series of medial-temporal lobe structures on both sides of the brain. These include the hippocampal and parahippocampal regions as well as the anterior cingulated cortex. Research in brain hemodynamics, electrophysiology, and magnetoencephalography has revealed the existence of different mechanisms involved in the two kinds of memory at the neuronal and molecular levels [12].

Perruchet and Poulin-Charonnat [2] suggest that, once they have learned a basic vocabulary set, children extract from parental input and memorize frequently appearing sequences of words located within the same attentional focus [13]. It may be added that the extracts are taken from particularly meaningful and pragmatically relevant parental utterances, cognitively accessible as to the contents, often located at the beginning of the end or utterances that are finely tuned to the developmental language levels of the children. Tonic accent is well marked with a lengthening of the final part of the utterance which helps discourse segmentation and slows down its rhythm. Parental utterances addressed to younger children are characterized by a slightly higher pitch that helps attracting and keeping the child's attention.

On this basis, mental representations isomorphous with the surface properties of the input are formed. In this model, the associative treatment of the language units is considered to be unconscious whereas the perceptions and mental representations may access consciousness. Perruchet and Poulin-Charonnat [2] claim that their model can account for behavioral adaptations usually described with systems of rules without having to appeal to a knowledge of these rules in the learners.

Longitudinal studies of verbal interactions between parents and language-learning children suggest a close relationship between adult utterances and the gradual lengthening and complexification of children's utterances. One may see on this point the longitudinal accounts of Moerk [14, 15, 16] and Rondal [17]; the entire corpus in the latter work being available in the Childes data bank [18].

A key learning variable is the frequency of appearance of the language aspects to be learned in the input. A statistical sensitivity has been documented in TD babies aged less than 12-month old [19]. A series of research works has documented frequency effects of parental input on the chronology of acquisition of a number of morphosyntactic structures [20; for a review and analysis]. For example, a significant negative correlation between order of acquisition and frequency of appearance of 
interrogative words in parental speech to their children has been found in several studies, explaining up to $36 \%$ of the chronological variance. Regarding grammatical morphology, Moerk [21], reanalyzing Brown's data [22], found statistically significant positive correlations between the frequency of appearance of six acoustically well-distinct grammatical morphemes in English (the articles $a$, the, the present progressive ingform, the Saxon genitive 's, prepositions in and on, and the regular past form -ed) in the transcripts between Eve's mother and Eve (one of the "Harvard children", recorded between 18 and 27 months; mean length of utterance (MLU) interval: 1,39 - 4,22). Brown had reported no correlation between input frequency and age of acquisition for the 14 grammatical morphemes that he studied, but he mixed in his counts the six acoustically well-distinct morphemes listed above with eight others acoustically less-distinct (e.g., regular plurals on nouns and auxiliaries) little represented in Eve's utterances and for which no clear correlation indeed existed between mother's input and Eve's productions. Several other series of research [20] have reported a clear effect of input frequency on the correct use of grammatical morphemes in various languages (e.g., genitive, dative, and accusative case marking in Polish).

High-frequency forms in the input not only favor the production of correct forms in children. They may cause errors where lower-frequency forms are the targets. This accounts for the well-known phenomenon of over-regularization of past tenses and noun plurals in children's language learning. Over-regularizations have often been judged to be the absolute proof of an early use of rules in language development. Typically, children's first productions of irregular morphology are usually correct. Slightly later, they start regularizing irregular forms; for example, producing blowed ${ }^{*}$ instead of blew, drinked* instead of drank, foots ${ }^{*}$ instead of feet, shelfs* instead of shelves). In a third phase, they return to the production of the correct forms. Frequency aspects of the input may account for these data without having to appeal to the use of particular rules. Regular forms generally correspond to high-frequency patterns in parental input. After repeated exposure children tend to over-regularize less frequently heard irregular forms. After a while, realizing that they are the only ones to produce such incorrect forms, they categorize verbs and nouns according to whether they exhibit regular or irregular morphological marking in the language. Matters may be slightly more complicated, however. One also needs taking into account the relative frequency of appearance of the correct and incorrect forms in the input. For example, it has been observed $[23,24]$ that the more frequent the irregular form the more likely children will produce this form as opposed to another less frequent irregular form (for example, in the examples given above, blew and feet are less likely to be over-regularized than drank and shelves likely because the former are higher-frequency forms). In the same way, children often produce two mouse* rather than the correct two mice because the latter form is less frequently found in the input. Conversely, they produce two foot ${ }^{*}$ more rarely probably because the correct form feet is more frequent in the input.

\section{A SEMANTIC RELATIONAL FRAMEWORK}

In my opinion, Perruchet and Poulin-Charonnat's model of implicit morphosyntactic learning [2], interesting as it is, needs completion on two major points. In the course of development and with more input exposure, even taking into account partial forgetting, the sheer number of sequences of words that are extracted by children from parental speech increases dramatically. It is not reasonable to assume that all the extracts could be stored verbatim at the crossroad between semantic and procedural memories and, consequently, that language production would amount to mobilizing relevant items in a repertoire of ready-to-be-expressed utterances. This would run against all we know about natural language functioning (e.g., speed of retrieval from devoted memory systems, generative character, creativity). Clearly, a reduction mechanism is needed equipped with a capacity to generate sequences of words organized according to the normative requirements of the tongue. As indicated, mainstream psycholinguistics considers that the grammatical categories of structural linguistics supply the system of abstractions needed for producing and understanding sentences. As mentioned also, this conviction is confronted with major difficulties even if it cannot be excluded completely.

I have suggested another abstraction mechanism [25]. As soon as the child has accumulated in memory a few instances of the more frequent word combinations in the input, the corresponding mental representations begin to involve meaning relations between words. These meaning relations (not to be confounded with lexical semantic or the meaning of individual words) are part of relational semantics (also called structural semantics). They include such cognitively accessible notions as agent of action, patient of action, entity, state, quality, possessor, 
instrument, location, benefit, etc., as well as their combinations (agent-action-patient, entity-quality, entity-location, action-instrument, etc.). Brown [22] has supplied a descriptive account of the development of these meaning relations in early language development. Along with cognitive development and language exposure, the relational semantic component of language becomes richer and more complex. There exists in the specialized literature several theoretical account of this language component. They more or less include the same dimensions often under different names. The most complete treatment is probably Chafe's contribution [26]. Very briefly summarized, Chafe's relational semantic system distinguishes four major types of verbs: action, state, process (e.g., Dishes are drying), and action-process (Mom dries the dishes), commanding corresponding types of nouns: agent, patient, entity. There are five secondary types of verbs commanding as many other semantic types of nouns: beneficiary (including possession), instrument, location, complement (an indication completing the meaning of a completable verb (for example, in Bread costs two dollars), and mental (including sensorial) experience (want, know, see, hear).

Meaning relations are optimally suited for constituting the basis of the needed reduction mechanism. They are in limited number, cognitively available from the beginning of development, abstract but of an abstraction level closer to the surface of the utterances as opposed to the linguistic formal categories, rules, and hierarchies. The latter are descriptively more economical but they have the inconvenient, from of psychological point of view, to be remote from utterance surface and to be less cognitively accessible than corresponding semantic categories. Once set in correspondence with the sequential patterns characteristic of the tongue, the semantic relations supply the patterns needed for generating all possible utterances in the language.

There is another aspect in which meaning relations can help organizing sentences. Perruchet-PoulinCharonnat's [2] model is based on extraction from the input and subsequent use of sequences of adjacent words. Meaning relations may also supply the basis for relating nonadjacent words in sentences. Current language production and reception/comprehension also encompass discontinuous propositional constituents; for example, in sentences with relative subordinates; e.g., The man who entered the rail station carried a strange parcel. In such sentences, the main clause The man carried a strange parcel is interrupted to make room for the relative one who entered the rail station (central embedding). Traditional account has it that language users need to access various hierarchical levels within a phrase structure grammar in order to understand sentences of that type. Alternatively language users may rely on a linearized conjunction of semantic relations. In the above example, the conjoined meaning relations would be: agent-action-patient and agent- action-location, with the first relation being interrupted to embed the second one but kept in short-term memory as a conceptual splint with regard to the second part of the sentence.

\section{MORPHOSYNTACTIC LEARNING IN COGNITIVE HANDICAP}

Difficulties in morphosyntactic functioning are the rule rather than the exception in genetic syndromes of cognitive handicap $[27,28]$. There appears to be partial syndrome specificity in the sense that the language problems may vary in gravity between syndromes and affect more certain language components than others $[29,30]$.

These difficulties still lack a specific explanation. An explanatory avenue that may be immediately rejected is in terms of a deficiency in the input addressed by parents to these children. It had been once proposed that the language input directed by parents to children with Down syndrome (DS) ${ }^{1}$ was too simplified and amounted to relative linguistic deprivation. Further research showed, however, that at corresponding developmental levels, the language addressed by parents of children with DS is not different from the language addressed by parents of TD children, neither in terms of morphosyntactic models or feedback contingent upon children's productions [31].

A possible explanation coming to mind is that the morphosyntactic difficulties in cognitive handicap are a direct consequence of the general cognitive limitation defining these conditions. As compelling as it may appear at first glance, this proposal turns out to be at least partially incorrect. It is true that there exists a loose correlation between severity of cognitive handicap and language development although the outcome may vary depending on the particular syndrome. Research shows, however, that cognitive handicap is not automatically tied to morphosyntactic difficulties. Hence it cannot be its main and even less its only causal factor. Relevant data are found in

${ }^{1}$ Etiology related to the presence of a supplementary complete or partial chromosome 21 in all or a part of the body cells. 
several inquiries [32, 33, 34, 35]. So-called languageexceptional adolescents and adults with moderate or even severe cognitive handicap were found to exhibit normal or normal-like productive and receptive morphosyntactic abilities. It follows that cognitive ability within normal psychometric limits per se is not a prerequisite for morphosyntacitc development. This does not mean, however, that particular cognitive variables (e.g., memory) could not play an important role in this development.

\section{IMPLICIT LEARNING IN COGNITIVE HANDICAP}

A limited amount of data has been published in recent years regarding the implicit learning of artificial grammar by participants with a cognitive handicap. A typical learning task in finite-state artificial grammar is as follows: an automaton generates sequences of item according to a few transitional rules. Participants are not informed of these rules or of the fact that there are rules commanding the transitions between items. They are requested to memorize the sequences of items as presented. In the test phase, participants are informed of the existence of transitional rules but they are not revealed the nature of these rules. They are presented with new sequences of items, some grammatical some ungrammatical and invited to differentiate between them. Results with TD participants generally show that the experimental subjects can sort the grammatical sequences from the ungrammatical ones as if they had discovered the transitional rules. But they demonstrate no conscious knowledge of these rules.

Several works with participants with mild cognitive handicap of various and mixed etiologies have been carried out to test the robustness of implicit learning and procedural memory across the spectrum of intellectual variability. The experimental protocols were adapted in various ways in order to better conform to the reduced cognitive abilities of the participant (see below for an illustration). Results suggest that implicit learning is a robust paradigm in the sense that its general principles apply to persons with cognitive handicap as well as to TD subjects. However, a number of differences have also been documented suggesting that the former subjects present limitations in some aspects of implicit learning.

A thorough investigation is that of Witt [36]. He compared a sample of 120 TD children divided in age groups between 5 and 8 years with a sample of children with cognitive handicap (of organic origin; etiologies not supplied in the report), intelligence quotient (IQ) between 50 and 70 points, chronological ages (CA) 9 years and 6 months to 10 years and 1 month, mental age (MA) 5 years and 8 months to 6 years and 4 months. The finite state automaton in Witt's series of experiments generated sequences of 3 , 4 or 5 colored flags representing teams of small pandas in a computerized video game of cord drawing presented in series of 8 sequences. Three types of series of colors were used: in one series, the sequential grammar allowed the successive repetitions of adjacent colors (e.g., blue-yellow-yellow; blueyellow-yellow -green; red-green-green-yellow-blue; and so on); in another series, the grammar allowed only the successive repetition of nonadjacent colors (e.g., blueyellow-blue; red-green-yellow-red; blue-yellow-greenyellow-blue; and so on). A third series presented the colors in random serial order and served as a control condition. In the test phase, the participants were informed that in the second day of the tournament, the organizers had forgotten to place the colors on the flags which, as a consequence, had remained white. Participants were invited to set series of 3,4 , or 5 colors on corresponding cardboards, selecting the colors one by one from a random color display. The instruction was simply to produce beautiful flags in a plausible attempt not to focus the participants' attention onto simply reproducing flags seen during the learning phase. The test phase was followed by a short debriefing session in the form of a pre-formulated and standardized questionnaire in which the experimenter asked the children whether $s($ he) knew why $s($ he) had been invited to play that particular game. The objective was to verify whether the participants had developed a conscious knowledge of the rules of the game and, if yes, whether they could verbalize them correctly.

Results show that children with a cognitive handicap demonstrate an ability to learn sequential relations implicitly. This indication attests to preserved capabilities of implicit learning in these children. It confirms previous reports with children, adolescents, young and aging adults with cognitive handicap with and without DS [37-40] and is congruent with Reber's [41] postulate regarding the robustness of basic aspects of the implicit learning paradigm across ages and IQ levels ${ }^{2}$. Witt's data are in opposition to the indications of Fletcher, Maybery and Bennett [42] suggesting that implicit learning would be largely inefficient below IQ 60 and/or an MA of 6 years. It

\footnotetext{
${ }^{2}$ Reber's conception is that implicit learning and memory represent evolutionary earlier biological systems compared to explicit and fully conscious ones, henceforth more likely to be present early in life and to be more resilient in front of possible cognitive pathologies.
} 
should be added, though, that Fletcher et al.'s experiments have been criticized on the ground of having too much of an explicit learning component. It is well known that persons with cognitive handicap practically always score lower than their TD peers in explicit learning tasks.

However, in Witt's experiment, children with cognitive handicap, contrary to their MA-matched TD peers, appeared to be sensitive only to adjacent repetitions of pairs of elements. No positional information was coded mentally. They appeared to have been restricted to identifying only the perceptually more prominent characteristics of the stimulus.

Another experimental paradigm, called visuomotor serial reaction times and involving implicit sequential learning, was used by Bussy, Charrin, Brun, Curie, and des Portes [43] with adolescents with Fragile-X syndrome $(F X S)^{3}$, DS, and TD children, all matched for MA. In this paradigm, a stimulus appears successively in one of several possible locations on a computer screen. Participants are requested to press a button upon detection of the stimulus and reaction times are measured. Unknown to the participants, there is a sequence involved in the succession of the stimuli. A decrease in reaction times is considered to be an indication that the subjects have detected a pattern in the sequential display even if they cannot verbalize the transitional rules. It was interesting to involve participants with FXS in the experiment given that they present anatomical abnormalities of the striatum, one of the basal ganglia involved in implicit memory. A statistically significant decrease in reaction times was recorded in the FXS group between the random block and some blocks with repeated sequences (not all, however, due to an important within group variability). In the DS group, the reaction times decreased significantly between the blocks with repeated sequences and the random ones except when there was an interfering random block located between two blocks with repeated sequences.

Desmottes, Meulemans, and Maillart [44] used a related research paradigm, called serial search task, with 24 TD children and 24 children with Specific Language Impairment $(\mathrm{SLI})^{4}$. These children, by definition, do no present an intellectual deficit. However, some of their difficulties in learning the

\footnotetext{
${ }^{3}$ In FXS, one gene (most often FMR-1, sometimes FMR-2) is mutated which reduces its transcription and results in a drastic reduction of the production of the developmentally needed proteins fmr-1 or fmr-2 in the brain.

${ }^{4}$ It is generally accepted that $\mathrm{SLI}$ is a genetic disorder related to the mutation of several genes not clearly identified. In particular, a mutation of the gene FOXP2 is strongly suspected.
}

morphosyntax of their mother tongue have been compared to the ones in cognitive handicap. TD and SLI children were matched for CA (7 to 12 years) and nonverbal IQ. Concrete bisyllabic words each illustrated by a picture were used. The pictures were presented in a $2 \times 2$ design on a computer screen and the arrangement of the four pictures varied for each trial. Children were instructed to locate the picture depicting each auditorily presented word with a touchscreen. Response time and accuracy were the dependent variables. After completing the last sequence learning task, an interview was made to probe a possible declarative knowledge of the sequence. Children then were informed of the existence of a regular sequence and requested to reproduce the sequence to which they had been exposed in a series of other trials. None of the children either TD or SLI demonstrated explicit knowledge of the sequence regularities. Data from the reaction times indicate that TD children implicitly learned both the spoken words as well as the motor sequences. In contrast a majority of children with SLI gave no indication of implicit learning of the sequence regularities in the motor as well that in the verbal task. These data support the hypothesis of a central (i.e., amodal) limitation in implicit sequence learning in most children with SLI but also of the existence of an important interindividual variability in the condition. The latter may explain some of the discrepancies in the results between comparable studies; for example, the one of Desmottes and colleagues and that Gabriel, Meulemans, Parisse, and Maillart [45] who reported similar sequence learning in visual and auditory serial reaction times in TD and children with SLI. Desmottes and colleagues also performed a series of correlational analyses between the serial search task performance and several tests assessing language development. A better performance on the serial search task was linked with better morphosyntactic and lexical abilities in TD children but only with lexical abilities in children with SLI. In another study using visuomotor serial reaction time, Desmottes and colleagues [46] observed that most children with SLI succeeded as well as TD children in the early acquisition stage of the sequence learning task. However, as training blocks progressed, only TD children improved their sequence knowledge while children with SLI did not appear to progress any further. Moreover, children with SLI failed to exhibit similar consolidations gains in sequence knowledge as TD children did at 24- hour and one-week time interval.

Returning to the genetic syndromes of cognitive handicap, a few studies have investigated the implicit learning ability of subjects with Williams syndrome 
(WS) ${ }^{5}$. Cashon, Ha, Graf Estes, Saffran, and Mervis [47] observed that babies with WS (aged between 8 and 20 months) demonstrate a spontaneous sensitivity to statistical properties of the input, such as the order of syllables in a continuous flow of artificial speech (for example, padotibidakutupiropadoti, etc.).

Schellenberg, Reber, DiGirolamo, and Wang [48] investigated implicit learning in 27 children and adults with WS matched for CA with TD subjects (ages between 9 and 50 years). They used a form of the standard artificial grammar learning paradigm. Results indicate that the WS subjects are able to implicitly learn the characteristics of a simple artificial grammar although at a lower level than TD persons.

Vicari [49] compared implicit and explicit memory processes in 12 children with WS, 14 with DS, and 32 TD children, all matched for MA (mean MA close to 6 years and 6 months in the three groups). Tests of verbal and visual implicit and explicit memory were administered (e.g., serial reaction time; a stem completion task in which the subjects had to complete words to which they had been exposed inadvertently before; free recall of a list of unrelated words). Children with DS and TD controls demonstrated comparable implicit memory abilities but TD subjects received higher scores in the explicit learning tasks. Children with WS demonstrated lower explicit memory abilities than TD controls. But they proved also less efficient in some implicit memory tasks particularly the serial reaction time.

What can we conclude from this review of an incomplete and still premature literature? It appears than persons with cognitive handicap are more impaired in explicit than in implicit learning. Several works suggest that persons with cognitive handicap are comparable to TD people in some of the experimental tasks used. It follows that general cognitive ability and $\mathrm{IQ}$ measures are not primary explanatory variables regarding implicit learning. The experimental tasks in implicit learning are artificial ones, carried out in artificial contexts. Obviously, the context of natural morphosyntactic development is different. Hopefully, however, we may extract some indications from the literature on artificial grammar and serial learning tasks in persons with cognitive handicap that would help us understanding better some of the limitations in natural morphosyntactic development in these subjects.

${ }^{5}$ This syndrome is etiologically linked to the absence of at least 18 genes on one of the two chromosomes 7.
A key indication is Witt's [36] demonstration that participants with (mild) cognitive handicap in opposition to their TD peers can implicitly learn sequential dependencies between adjacent units in pairs of stimuli but not nonadjacent ones or even adjacent dependencies beyond pairs of stimuli. In other words, the subjects with cognitive handicap can implicitly learn only simple relationships and relationships that are perceptually closer and located within shorter attention spans. They also appeared unable to encode positional information regarding items in visual displays. Data collected by Bussy et al.[43] are convergent with Witt's indications, as they found that even if DS adolescents could implicitly learn sequential relations in a serial reaction time (but not MA-matched adolescents with FXS), the learning seemed to vanish to a large extent when a block with random presentation of the stimuli was placed in between blocks with repeated sequences. This suggests a weakness in maintaining the relevant traces in implicit learning and resisting distractors. Cashon et al. [47] found that a basic statistical sensitivity to auditory stimuli is present in babies with WS which may be considered to be a prerequisite for implicit language learning. Schellenberg et al. [48] found their subjects with WS (children, adolescents, and adults) able to learn implicitly an artificial grammar but at a lower level of performance than TD persons matched for CA with the WS subjects. Vicari [49], however, found his children and adolescents with WS to perform lower on a series of implicit learning tasks than MA-matched TD children and children with DS.

\section{MORPHOSYNTACTIC DIFFICULTIES IN DOWN SYNDROME}

As the expressive and receptive morphosyntactic problems of persons with Down syndrome have been the most thoroughly studied so far, it is relevant to start with a brief summary of these problems [3] before analyzing them from the standpoint of implicit learning and its possible limitations in cognitive handicap. I am not suggesting that other limitations should not be taken into account. Phonological memory, for example, is of great importance in itself and probably interacts with implicit learning of morphosyntax (particularly grammatical morphology). One may see Conners [50] for a discussion of this question.

Regarding the syntactic regulation of language in DS, one generally observes: (1) A lesser comprehension and reduced and unstable use of articles, prepositions, auxiliaries, copulas, pronouns, 
and conjunctions even when DS subjects are matched with TD peers for MA; (2) No syntactic comprehension of reversible passives; (3) No comprehension of temporal sentences with clause order not matching order of events; (4) Fewer reversals of order of grammatical subject and copula or auxiliary verb in interrogative sentences; and (5) Reduced use and limited understanding of longer sentences with coordinated clauses and overall subordinated ones.

As to grammatical morphology in DS, one usually witnesses: (1) A reduced use of verb obligatory inflections. Frequent omissions include contracted will, contracted am, contracted is, third-person singular inflection of regular verbs, regular past-tense -ed endings, present progressive -ing endings, noun plural -s endings, and noun possessive endings; (2) Unstable marking of number on nouns; (3) Omission of number agreement between grammatical subject and conjugated verb.

As indicated, there seems to be partial specificity between major syndromes of cognitive handicap. For example, children and adolescents with WS are usually described as presenting a better morphosyntactic development than people with other genetic syndromes of cognitive handicap. It may be justified to link these indications to anatomical peculiarities in these syndromes [51]. For example, people with WS and SD have similar degrees of microcephaly, cerebral hypoplasia, brain volumetric reduction, and reduced level of axon myelination particularly in brain associative areas. Synaptic density is reduced in both syndromes and many synapses have abnormal morphology and contacts points. These anomalies are a consequence of abnormal neurogenesis during pre-, peri-, and postnatal periods of development. However, important brain differences between the two syndromes have been identified. The brains of the persons with SW are characterized by a relative preservation of the anterior and median parts in spite of an important reduction of the parietal and occipital areas. This cerebral morphology is compatible with the better linguistic abilities in this syndrome as well as with a reduced ability in spatial cognition.

The brain of persons with DS is characterized by a marked underdevelopment of the frontal and temporal areas (hosting two of the two major brain language areas, i.e., the Broca and the Wernicke areas). This anatomical profile corresponds to the reduction in verbal fluency, tendency to perseverate, and the difficulties in executive functions that are well spread in this syndrome. Other anatomical differences have been documented between people with SW and SD at the level of the basal ganglia and the cerebellum. The cerebellum volume is about at the norm in SW (however, there is a reduction of the excitatory neurotransmitter $\mathrm{N}$-acetylasparate) but it is reduced down to $70 \%$ in SD. It is known that this structure (particularly the neocerebellum) is involved in several aspects of morphosyntactic functioning, particularly the sequencing aspects. Such is also the case for the basal ganglia with respect to implicit procedural learning. Persons with SW typically present an important atrophy and volumetric reduction of the striatum, one of the nuclei at the basis of the brain, much involved in implicit learning and memory. Such a reduction does not seem to exist in DS.

Children with DS in their vast majority do develop linguistically, including in basic morphosyntactic abilities (for example, word order in short utterances is usually correct). This development is incomplete, however, and does not extend to more sophisticated structures. The question is why. Assuming that morphosyntactic development proceeds mostly implicitly and given that these persons do indeed acquire the basic aspects of the morphosyntax of their maternal tongue, how is it that most of the time they do not seem to advance much beyond that stage?

Perceptual apprehension of sequential units in the input is involved in implicit learning. Is it typically deficient in children with DS? Visual perceptual deficits have been reported in persons with cognitive handicap including DS [52, 53]. In DS, a mild to moderate auditory deficit mostly of the transmission type touches a minority of children. However, it is doubtful that these problems could be serious enough as to impede or gravely impair morphosyntactic processing although in some cases they could limit treatment to more prominent units in the input.

No major global attentional deficit has been reported in cognitive handicap [54]. In DS, however, sustained attention for visual stimuli is better preserved than for auditory ones [55]. A particular weakness in reducing attentional focus to more limited sequence of units in the input may be involved in the restricted data processing demonstrated by these subjects.

It does not seem reasonable to suspect a particular deficiency in persons with DS and/or other genetic syndromes of cognitive handicap in basic associative ability. The indication of Cashon et al. [47] that babies 
with WS are sensitive to simple sequential patterns would run against such a hypothesis. The fact, in Witt's data [36], for example, that subjects with cognitive handicap were able to learn to associate simple adjacent items is another positive indication in the same respect. And preserved basic word order in simple sentences in persons with DS and other genetic syndromes of cognitive handicap is yet another indication going in the same interpretive direction.

However, relating units in longer utterances including nonadjacent ones and constructing appropriate mental representations bearing on these relations is another matter. Well-documented limitations in auditory short-term (working) and longerterm memory in DS and related syndromes [50, 56, 57] render less efficient the process of encoding relevant incidental information and recollecting it for later use [58].

As mentioned, I have suggested [25] that in TD children the meaning relations holding between words in utterances play a major role in morphosyntactic development.

When they begin to combine two and three words within the same utterance, children with cognitive handicap, including those with moderate and even severe retardation, appear to express the same range of relational meanings as reported in language development of TD children. They also understand correctly the same set of basic relational meanings when they are realized in the speech of other people [59, for a review]. However, children with cognitive handicap have particular difficulties in combining semantic meanings beyond simple associations and it is likely that they fall short of being able to make full use of relational semantics in more advanced aspects of morphosyntactic development.

Let us analyze the major morphosyntactical limitations in Down syndrome as summarized above. A difficulty with reversible passives (e.g., The car was hit by the truck) testifies to an inability to realize that specific marks in sentence surface (i.e., use of auxiliary, verb in past-participle form, and use of agentive preposition) signal that the semantic relation agent-action-patient while still holding has to be decoded in the reverse way (patient being expressed first). Temporal sentences with clause order not matching factual order of events (e.g., He left after eating as opposed to $\mathrm{He}$ ate and left) raise a comparable problem. The production of the conjunction after informs the listener that the first part of the sentence (corresponding to a meaning relation agentintransitive action) is not the one having occurred first in reality. In order to understand correctly sentences of that kind, one must pay particular attention to this conjunction that is not placed in front of the sentence hence the procedural difficulty.

Difficulties with interrogative reversals of first verbal element and grammatical subject may in part reflect a negative frequency effect from the input tied to the relative reduction of such forms in contemporary English (common language now favoring noninverted interrogative forms). It is also probably linked to the uneasiness of persons with DS in dealing with inverted forms and the necessity to perform additional coding or decoding to manage them.

The difficulties in DS with articles, prepositions, and conjunctions have to do with two things: grasping the particular relational meaning involved in the correct use of these forms and managing their syntactic ordering in utterances. Articles must be placed before nominal entities. They carry two semantic distinctions: a number one (singular vs plural) usually easily understood by all children; and another one between indefinite and definite reference (i.e., a reference already specified in the discourse or from nonlinguistic context) that is more difficult to understand.

The prepositions command prepositional phrases that are ordered relatively rigidly. Major problems are with the semantics of the prepositions. Each one expresses a particular meaning relation (location - in, on -, origin - from -, destination - to -, accompaniment with -, possession - of -, saxon genitive's -, instrument by -, etc.) which need to be clearly understood before any possible proper use. The same is true of conjunctions but this time at the level of sequences of clauses in complex sentences. A proper use implies that relations of union (and), opposition (or), negation (neither), consequence (then) etc., be correctly understood in coordinated clauses, and relations of time, cause, condition, circumstance, comparison, etc. (when, after that, because, if, given that, as, etc.) in sentences with subordinated clauses. Another cause for the difficulties with these structures is an increased length of the corresponding sentences and the fact that they may include discontinuous constituents (for example, central embedding in sentences with relative clauses).

Personal, relative, interrogative, possessive, demonstrative and indefinite pronouns are lexical 
substitutes for nouns in order to avoid renaming entities that may be identified from the nonlinguistic context, or have already been introduced in the verbal exchange. Substitutes must exemplify a cued relationship with the substituted entity. This may be a particular marking for person, gender, number, proximity vs distance (real or abstract), and/or position in the sentence, or in a sequence of sentences (e.g., pronominal anaphora). Understanding and expressing such a relationship between pronoun and substituted noun is often a difficult challenge for persons with DS probably because it implies a close monitoring of the sentence, and/or the extralinguistic context (immediate or more remote), and an ability to understand and express the relevant marks.

As to grammatical morphology, major problems are found in understanding and producing inflections marking number on nouns, person, time, and mode on verbs, and in expressing number agreement between grammatical subject and verb. They concern the expression of particular semantic notions through specific marking and often (but not only) distal relations between words in the sentence (for example, plural reference in the first nominal phrase determining plural marking on the main verb following a few words later). As indicated, distal and discontinuous associations often make difficulties for the persons with a cognitive handicap for reason of limited attentional focus and short-term memory, and difficulties in sentence monitoring. There may also be processing overweight with longer sentences in these people who may be at a major disadvantage when having to deal simultaneously with the several subtasks involved in language production (recalling and organizing contents mentally, mobilizing pragmatic indexation and semantic matrix, selecting lexical units, linearizing expression according to the sequential patterns of the tongue, properly inflecting the words that need to be inflected, and marking the necessary agreements between words).

\section{INTERVENTION PERSPECTIVE}

From the preceding review and discussion, several basic considerations have emerged regarding morphosyntactic development and functioning in persons with cognitive handicap and particularly those with DS and related genetic conditions. What recommendations can be made on such a basis for intervention with these children and adolescents?

Intervention by definition is carried out in an explicit manner, i.e., subjects are proposed various activities to be executed purposively with the assistance of a clinician. This may appear somewhat in contradiction with the fact that spontaneous morphosyntactic development takes place mostly implicitly. But there is no other way to remediate to the insufficiency of natural development in persons with cognitive handicap.

An explicit morphosyntactic intervention in Down syndrome and related conditions should concentrate on the following targets: promoting understanding and expression of advanced semantic relations, increasing attentional focus, developing positional knowledge regarding words in sentences, developing sensitivity regarding nonadjacent units and discontinuous constituents in sentences, and promoting understanding and use of coordinated phrases and clauses and of subordinated clauses in complex sentences.

A computer equipped with adequate programs can be of great help in morphosyntactic remediation. Two aspects deserve particular attention. At the level of the meaning relations, animated computer displays including arrows and other pointing subsystems can be useful in assisting subjects in constructing mental representations and memories of more complex meaning relations holding between entities and corresponding words. At the sentence level, animated computer programs using arrows, colors, and other pedagogical devices, can help subjects identifying semantic/syntactic patterns in sentences, including relationships between nonadjacent units and discontinuous constituents, and stressing authorized displacements of constituents. The semantic and formal relationships between words in sentences that need to be understood in order to comply with tongue requirements in matter of grammatical morphology can also be demonstrated using computer technology. Visual programs can be synchronized with vocal synthesis in order to attract attention, favor lexical encoding, and stimulate auditory memory.

\section{REFERENCES}

[1] Rondal JA. L'apprentissage implicite du langage. Wavre, Belgium: Mardaga 2011.

[2] Perruchet $P$, Poulin-Charonnat B. The learnability of language. Insights from the implicit learning literature. In: Rebuschat $\mathrm{P}$, editor. Implicit and explicit learning of languages. Amsterdam: Benjamins 2015; 139-65. https://doi.org/10.1075/sibil.48.07per

[3] Rondal JA, Guazzo GM. Morphosytactic difficulties and rehabilitation in persons with Down syndrome. In: Hodapp R, editor. International review of research in developmental disabilities. New York: Elsevier 2012; pp. 85-107. 
[4] Rondal JA. Une théorie du développement et du fonctionnement morphosyntaxique. Paris: L'Harmattan 2014.

[5] Rondal JA. Natural morphosyntax. The case for implicit surface learning and processing. Cogn Linguist Stud 2015; 2: 181-204.

https://doi.org/10.1075/cogls.2.2.01ron

[6] Stromswold K. The heritability of language: A review and meta-analysis of twin, adoption, and linkage studies. Language 2001; 77: 647-723. https://doi.org/10.1353/lan.2001.0247

[7] Gazzaniga M. Human. New York: Harper Collins 2008.

[8] Dominey $\mathrm{P}$, Hoen $\mathrm{M}$, Blanc $\mathrm{J}$, Lelekov-Boissard $\mathrm{T}$. Neurological basis of language and sequential cognition: Evidence from simulation, aphasia, and ERP studies. Brain Lang 2003; 86: 207-25. https://doi.org/10.1016/S0093-934X(02)00529-1

[9] Osterhout L, Kim A, Kuperberg G. The neurobiology of sentence comprehension. In: Spivey M, McRae K, Joanisse $M$, editors. The Cambridge handbook of psycholinguistics. Cambridge, UK: Cambridge University Press 2012; pp. 36589.

https://doi.org/10.1017/CBO9781139029377.025

[10] Ullman M. Contributions of memory circuits to language: The declarative/procedural model. Cognition 2004; 92: 231-70. https://doi.org/10.1016/j.cognition.2003.10.008

[11] Turk-Browne N, Yi D, Chun M. Linking implicit and explicit memory: Common encoding factors and shared representations. Neuron 2006; 49: 917-927. https://doi.org/10.1016/j.neuron.2006.01.030

[12] Kandel E. In search of memory. New York: Norton 2005.

[13] Bannard C, Mattews D. Stored word sequences in language learning: The effect of familiarity on children's repetitions of four-word combinations. Psychol Sci 2008; 19: 241-8. https://doi.org/10.1111/j.1467-9280.2008.02075.x

[14] Moerk E. The mother of Eve as a first-language teacher. Norwood, NJ: Ablex 1983.

[15] Moerk E. First language taught and learned. Baltimore, MD: Brookes 1992.

[16] Moerk E. The guided acquisition of first language skills. Stamford, CT: Ablex 2000.

[17] Rondal JA. Comment l'enfant apprend la grammaire de sa langue sans le savoir. Paris: Edilivre 2014.

[18] MacWhinney B. The Childes project. Tools for analyzing talk. Hillsdale, NJ: Erlbaum 1994.

[19] Denison S, Xu F. The origin of probabilistic inference in human infants. Cognition 2014; 130: 335-47. https://doi.org/10.1016/j.cognition.2013.12.001

[20] Ambridge B, Kidd E, Rowland C, Theakston A. The ubiquity of frequency effects in first language acquisition. J Child Lang 2015; 42: 239-73. https://doi.org/10.1017/S030500091400049X

[21] Moerk E. Relationships between parental input frequencies and children's language acquisition: a reanalysis of Brown's data. J Child Lang 1980; 7: 105-18.

https://doi.org/10.1017/S0305000900007054

[22] Brown R. A first language. The early stages. Cambridge, MA: Harvard University Press 1973.

[23] Maslen R, Theakston A, Lieven E, Tomasello M. A dense corpus study o past tense and plural over-regularizations in English. J Speech Lang Hear Res 2004; 47: 1319-33. https://doi.org/10.1044/1092-4388(2004/099)

[24] Matthews D, Theakston A. Errors of omission in Englishspeaking children's production of plurals and the past tense: The effects of frequency, phonology, and competition. Cogn Sci 2006; 30: 1027-52. https://doi.org/10.1207/s15516709cog0000 66
[25] Rondal JA. Peut-on se dispenser des catégories grammaticales et des règles formelles dans l'explication de l'acquisition de la syntaxe en langue naturelle? Submitted for publication.

[26] Chafe W. Meaning and the structure of language. Chicago: University of Chicago Press 1973.

[27] Chapman R. Language and communication in individuals with Down syndrome. In: Abbeduto L, editor. International review of research in mental retardation. New York, NY: Academic 2003; pp. 1-34. https://doi.org/10.1016/s0074-7750(03)27001-4

[28] Rondal JA. Psycholinguistique du handicap mental. Marseille, France: Solal 2009.

[29] Rondal JA, Hodapp R, Soresi S, Dykens E, Nota L. Intellectual disabilities. Genetics, behaviour and inclusion. London: Whurr 2004.

[30] Rondal JA, Perera J, Eds. Down syndrome. Neurobehavioural specificity. London: Whurr 2006.

[31] Rondal JA, Docquier L. Maternal speech to Down syndrome children. An update. J Speech Lang Pathol Appl Behav Anal 2006; 1: 218-27.

https://doi.org/10.1037/h0100197

[32] Curtiss $\mathrm{S}$. Abnormal language acquisition and the modularity of language. In: Newmeyer F, editor. Linguistics: The Cambridge survey. Cambridge, UK: Cambridge University Press vol. 2: pp. 96-111.

[33] Yamada J. Laura: A case for the modularity of language. Cambridge, MA: Massachusetts Institute of Technology Press 1990.

[34] Rondal JA. Exceptional language in Down syndrome. Implications for the cognition-language relationship. New York: Cambridge University Press 1995. https://doi.org/10.1017/CBO9780511582189

[35] Smith N, Tsimpli I. The mind of a savant: Language learning and modularity. Oxford, UK: Blackwell 1995.

[36] Witt A. L'apprentissage implicite d'une grammaire artificielle chez l'enfant avec et sans retard mental: rôle des propriétés du matériel et influence des instructions. Thèse de Doctorat en Psychologie. Dijon, France: Université de Bourgogne. NNT HAL Open archives: 20010DIJOL019; 2011.

[37] Wyatt B, Conners F. Implicit and explicit memory in individuals with mental retardation. Am J Ment Retard 1998; 102: 511-26.

https://doi.org/10.1352/08958017(1998)102<0511:IAEMII>2.0.CO;2

[38] Atwell J, Conners F, Merrill E. Implicit and explicit learning in Young adults with mental retardation. Am J Ment Retard 2003; 108: 55-68.

https://doi.org/10.1352/08958017(2003)108<0056:IAELIY > 2.0.CO;2

[39] Vinter A, Detable C. Implicit learning in children and adolescents with mental retardation. Am J Ment Retard 2003; 108: 94-107.

https://doi.org/10.1352/08958017(2003)108<0094:ILICAA $>2.0$. CO;2

[40] Krinsky-McHale S, Devenny D, Kitter P, Silverman W. Implicit memory in aging adults with mental retardation with and without Down syndrome. Am J Ment Retard 2003; 108: 21933.

https://doi.org/10.1352/08958017(2003)108<219:IMIAAW >2.0.CO;2

[41] Reber A. Implicit learning and tacit knowledge: An essay on the cognitive unconscious. New York: Oxford University Press 1993.

[42] Fletcher J, Maybery M, Bennett S. Implicit learning differences: A question of developmental level? J Exp Psych [Learn Mem Cogn] 2000; 26: 246-52.

https://doi.org/10.1037/0278-7393.26.1.246 
[43] Bussy G, Charrin E, Brun A, Curie A, des Portes V. Implicit procedural learning in Fragile $X$ and Down syndrome. $J$ Intellect Disabil Res 2011; 55: 521-8. https://doi.org/10.1111/j.1365-2788.2011.01410.x

[44] Desmottes L, Meulemans T, Maillart, C. Implicit spoken words and motor sequences learning are impaired in children with Specific language Impairment. J Int Neuropsych Soc 2016; 22: 520-29. https://doi.org/10.1017/S135561771600028X

[45] Gabriel A, Meulemans T, Parisse C, Maillart C. Procedural learning across modalities in French speaking children with specific language impairment. Appl Psycholing 2014; 36: 747-69. https://doi.org/10.1017/S0142716413000490

[46] Desmottes L, Meulemans, L, Maillart C. Later learning stages in procedural memory are impaired in children with Specific Language Impairment. Res Dev Disabil 2016; 48: 53-68. https://doi.org/10.1016/j.ridd.2015.10.010

[47] Cashon C, Ha O, Graf Estes K, Saffran J, Mervis C. Infants with Williams syndrome detect statistical regularities in continuous speech. Cognition 2016; 154: 165-168. https://doi.org/10.1016/j.cognition.2016.05.009

[48] Schellenberg EG, Reber A, DiGirolamo K, Wang P. Implicit learning in children and adults with Williams syndrome. Dev Neuropsych 2003; 23: 201-25. https://doi.org/10.1080/87565641.2003.9651892

[49] Vicari S. Implicit versus explicit memory function in children with Down and Williams syndrome. Down Synd Res Pract 2001; 7 (1): 35-40. https://doi.org/10.3104/reports.112

[50] Conners F. Phonological working memory difficulty and related interventions. In: Rondal JA, Buckley S, editors. Speech and language intervention in Down syndrome. London: Whurr 2003; pp. 31-49.
[51] Rondal JA. Psycholinguistique du handicap mental. Marseille, France: Solal 2009.

[52] Fox R, Oross S. Perceptual deficits in mildly retarded adults. In: Bray N, editor. International review of research in mental retardation. New York: Basic Books 1992; pp. 1-27. https://doi.org/10.1016/s0074-7750(08)60114-7

[53] Wang Y, Chang C, Chen S, Wang, C, Wuang Y. Profiles of visual perceptual functions in Down syndrome. Res Dev Dis 2015; 37: 112-118. https://doi.org/10.1016/j.ridd.2014.11.008

[54] Burack J, Evans D, Klaiman C, Iarocci G. The mysterious myth of attention deficits and other defect theories: Contemporary issues in the developmental approach to mental retardation. In: Glidden L, editor.International review of research in mental retardation. San Diego, CA: Academic Press 2001; pp. 299-320.

[55] Faught G, Conners F, Himmelberger Z. Auditory and visually sustained attention in Down syndrome. Res Dev Dis 2016; 53-54: 135-146. https://doi.org/10.1016/j.ridd.2016.01.021

[56] Carlesimo G, Marotta Vicari S. Long-term memory in mental retardation: Evidence for a specific impairment in subjects with Down's syndrome. Neuropsychologia 1997; 35: 71-9. https://doi.org/10.1016/S0028-3932(96)00055-3

[57] Rondal JA. La rehabilitation des personnes porteuses d'une trisomie 21. Suivi medical, neuropsychologie, pharmacothérapie et thérapie génétique. Paris: L'Harmattan 2013.

[58] Witt A, Vinter A. Children with intellectual disabilities may be impaired in encoding and recollecting incidental information. Res Dev Disabil 2013; 864-71. https://doi.org/10.1016/j.ridd.2012.11.003

[59] Rondal JA, Edwards S. Language in mental retardation. London: Whurr 1997.

DOI: https://doi.org/10.6000/2292-2598.2017.05.01.4

(c) 2017 Jean Adolphe Rondal; Licensee Lifescience Global.

This is an open access article licensed under the terms of the Creative Commons Attribution Non-Commercial License (http://creativecommons.org/licenses/by-nc/3.0/) which permits unrestricted, non-commercial use, distribution and reproduction in any medium, provided the work is properly cited. 